\title{
Estudo de caso utilizando mapeamento de prospecção tecnológica como principal ferramenta de busca científica
}

Keize Katiane dos Santos Amparo

\author{
Centro Integrado de Manufatura e Tecnologia \\ (SENAI CIMATEC)
}

Maria do Carmo Oliveira Ribeiro

Centro Integrado de Manufatura e Tecnologia (SENAI CIMATEC)

Lílian Lefol Nani Guarieiro

Centro Integrado de Manufatura e Tecnologia (SENAI CIMATEC). INCT de Energia e Ambiente

Este trabalho apresenta conceitos e metodologias para a realização de estudos de prospecção, utilizando o documento de patente, com fonte de informação tecnológica, para verificar o estado da arte de sistemas denominados "Túnel de diluição", para estudos de emissões veiculares. Este estudo visa a demonstrar, dentre outras, a importância da gestão da informação, como mecanismo de apoio ao monitoramento tecnológico para tomada de decisão, tendo como vetor principal a análise de informação tecnológica em documentos de patentes e o papel do profissional da informação, utilizando as metodologias de estratégias de busca em bancos e bases de dados, como matéria-prima para a realização dos estudos de prospecção. Os métodos de prospecção tecnológica utilizados foram feitos, inicialmente, através de buscas de anterioridade e similaridade em bancos de dados de patentes, nacionais e internacionais, com estratégias de buscas adequadas aos recursos disponíveis em cada base de dados. Assim, este trabalho apresenta a fase da inteligência competitiva, na qual são identificadas, mapeadas, coletadas, tratadas e analisadas as informações extraídas de bases de patentes para geração de mapas de conhecimento, como subsídio ao pensar estratégico, objetivando facilitar e apoiar a tomada de decisão na direção do desenvolvimento do 
setor industrial. Com os resultados alcançados, foi possível identificar o percentual de documentos de patentes depositados pela área acadêmica, empresas, instituições de pesquisa e pessoas físicas, além de identificar as rotas tecnológicas e empresas detentoras da referida tecnologias no mundo.

Palavras-chave: Prospecção tecnológica; Estudos prospectivos; Patentes; Informação tecnológica; Ciência da Informação.

\section{Case study using mapping technology foresight as the main tool of scientific research}

This paper presents concepts and methodologies for conducting research studies using the patent document with source of technological information to verify the state of the art system called "Dilution Tunnel" for studies of vehicle emissions. This study aims to demonstrate, among other things, the importance of information management as a mechanism to support technology monitoring for decision making, with the main vector analysis of technological information in patent documents and the role of the information professional using the methodologies search strategies in banks and databases as raw material for the studies of prospecting. Technological forecasting methods were initially used by prior art searches and similarity in patent databases, national and international, as search strategies appropriate to the resources available in each database. This work presents the stage of competitive intelligence where they are identified, mapped, collected, processed and analyzed the information extracted from patent databases to generate knowledge maps as an aid to strategic thinking aimed at facilitating and supporting decision making toward the development of the industrial sector. With the results obtained it was possible to identify the percentage of patent documents deposited by academic, business, research institutions and individuals, and to identify the technological routes and holding companies of such technologies in the world. 
Keywords: Technology foresight; Prospective studies; Patents; Information technology; Information Science.

Recebido em 02.10.2012 Aceito em 10.12.2012

\section{Introdução}

Nas últimas décadas, o mundo passou por uma revolução radical, com impactos nas áreas política, econômica, tecnológica e social. Para os países que não atingiram o nível de desenvolvimento desejado, esses impactos levaram a um crescente distanciamento em relação aos países considerados de "primeiro mundo". A visão orientada para o futuro é o caminho rumo a uma melhor sustentabilidade e fortalecimento da capacidade do país para aproveitar as oportunidades futuras em uma economia globalizada. Na área tecnológica, essencial para qualquer projeto de desenvolvimento do país, a necessidade de estudos prospectivos é evidente. As mudanças tecnológicas, ocorridas nas duas últimas décadas, indicam que ainda temos um longo caminho para percorrer, o que torna necessária a utilização de caminhos alternativos para orientar o futuro. Os estudos de prospecção tecnológica é um desses caminhos.

O termo prospecção tecnológica designa atividades de prospecção centradas nas mudanças tecnológicas, em mudanças na capacidade funcional ou no tempo e significado de uma inovação. Visa a incorporar informação ao processo de gestão tecnológica, tentando predizer possíveis estados futuros da tecnologia ou condições que afetam sua contribuição para as metas estabelecidas. Castells (1999) defende a tese de que o surgimento da economia da informação caracteriza-se pelo desenvolvimento de uma nova lógica organizacional baseada na convergência e na interação entre as tecnologias de informação e comunicação (TIC), os modelos de gestão e as articulações crescentes de redes de pessoas e empresas. Contudo, entre a decisão estratégica de investir e a apropriação dos ganhos de competitividade resultantes, há um grande número de etapas, nas quais, o ponto crítico é a incerteza. Para Coelho (2003), a prospecção de tecnologia, por meio da gestão de informação, é extremamente útil para apresentar o estado-da-arte de determinada área tecnológica, com o objetivo de gerar informações sobre a sua trajetória passada e sobre as tendências de mercado e percepção de sinais fracos.

Inovar nos métodos e processos de gestão é, portanto, um dos desafios face às exigências de um mercado globalizado, com concorrência acirrada, elevado grau de incertezas e um grande volume de informação disponível no mundo. O processo ou ciclo do Sistema de Inteligência se baseia na identificação precisa das necessidades do usuário e de sua adequação às orientações estratégicas. Essas são condicionantes importantes à eficácia do processo, principalmente no que tange à gestão da informação, ou seja, na busca, seleção, tratamento da informação de 
interesse para permitir, dentre outras, a visualização de tendências e subsídios ao processo decisório.

O tratamento automático da informação se baseia em métodos bibliométricos, ou seja, método matemático e estatístico que permite medir a comunicação escrita, comportando as leis de Bradford, Lotka e Zipf (ALVARADO, 1984). No contexto da inteligência competitiva, a base conceitual de Zipf, que descreve a frequência no uso de palavras em um determinado texto, permite agregar valor na análise de conteúdos informacionais expressivos e agilizar o processo de interpretação de dados.

Nesse sentido, os estudos de Prospecção Tecnológica são de fundamental importância e constituem a ferramenta básica para orientar os esforços empreendidos para o desenvolvimento de tecnologias. Hoje, eles são componentes fundamentais como subsídios para ampliar a capacidade de antecipação e estimulam a organização dos sistemas de inovação, não somente no âmbito empresarial, mas, também, no meio acadêmico. As mudanças tecnológicas, ocorridas nas duas últimas décadas, indicam que ainda virão muitas inovações, o que torna necessária a compreensão das forças que orientarão o futuro.

A gestão da informação, associada aos avanços das tecnologias, vem sendo cada vez mais valorizada e seu uso sistemático entendido como investimento face ao potencial de agregação de valor e geração de novos saberes (CANONGIA et al., 2004). Para Godet et al. (2000), toda forma de prever ou predizer o futuro é uma impostura, porque o futuro é fruto da vontade humana, não está escrito em parte alguma, sendo necessário construí-lo.

O objetivo dos estudos de prospecção não é desvendar o futuro e, sim, delinear e testar visões possíveis e desejáveis para que, hoje, sejam feitas escolhas que contribuirão, de forma mais significativa, na construção do futuro. Por outro lado, as metodologias de prospecção são ferramentas que buscam entender as forças que orientam o futuro, visando à construção do conhecimento.

De acordo com Kupfer e Tigre (2004), Prospecção Tecnológica pode ser definida como um meio sistemático de mapear desenvolvimentos científicos e tecnológicos futuros capazes de influenciar de forma significativa uma indústria, a economia ou a sociedade como um todo. Diferentemente das atividades de previsão clássica, que se dedicam a antecipar um futuro suposto como único, os exercícios de prospecção são construídos a partir da premissa de que são vários os futuros possíveis.

Os métodos de Prospecção Tecnológica são usados há várias décadas em diversos países, como uma ferramenta para orientar os esforços empreendidos para a pesquisa, desenvolvimento e inovação, PD\&I. Conforme Antunes (2002), os registros iniciais de utilização sistematizada das informações como ferramenta estratégica são datados da década de 50, cujo objetivo principal era a redução do tempo entre a invenção e a disposição dos produtos novos no mercado e, a essa atividade, deu-se o nome de Prospecção Tecnológica. 
A partir da década de 80, diversos autores perceberam que 0 fenômeno da inovação implicava em mudanças nos métodos e propósitos da Prospecção Tecnológica. Desde então, foram propostas novas metodologias e surgiram novos termos e definições para os estudos de prospecção e a terminologia comumente utilizada inclui as seguintes expressões "Technology Foresight", "Forecasting", "Prospective Studies", "Prospective Studies", "Future Research", "Futuribles", entre outros.

Bahruth et al. (2006 apud Mayerhoff 2008) mencionam que os estudos de prospecção tecnológica constituem quatro fases distintas: 1) fase preparatória para definição de objetivos, escopo, abordagem e a metodologia utilizada durante a prospecção; 2) fase pré-prospectiva, na qual é realizado o detalhamento da metodologia, bem como o levantamento da fonte de dados; 3) fase prospectiva, que se refere à coleta, ao tratamento e à analise dos dados, obtidos durante a fase préprospectiva; e 4) fase pós-prospectiva, que é a etapa final do processo e inclui a comunicação dos resultados, bem como a implementação das ações e o monitoramento

Kupfer e Tigre (2004) descrevem que os métodos de Prospecção Tecnológica podem ser classificados em três grupos: 1) monitoramento (Assessment), que consiste no acompanhamento sistemático e contínuo da evolução dos fatos e na identificação de fatores portadores de mudança; 2) previsão (Forecasting), que consiste na realização de projeções baseadas em informações históricas e modelagem de tendências; e 3) visão (Foresight), que consiste na antecipação de possibilidades futuras, com base em interação não estruturada entre especialistas.

$\mathrm{Na}$ fase do monitoramento, as fontes de informação devem ser identificadas, as informações devem ser coletadas, analisadas e estruturadas para o uso. O monitoramento não é uma técnica de prospecção, mas é a mais básica e amplamente utilizada, porque prevê o pano de fundo necessário, no qual a prospecção se baseia (DA SILVA, 2006).

Como a informação tecnológica é um dos requisitos indispensáveis para a realização de estudos de prospecção para a área acadêmica e científica, ela deve ser obtida através de fontes confiáveis e contínuas. Sendo assim, Aguiar (1991) define a informação tecnológica como "todo tipo de conhecimento relacionado com o modo de fazer um produto ou prestar um serviço para colocá-lo no mercado".

Os estudos de prospecção, que necessitam da informação tecnológica, podem ser encontrados nos bancos de dados de patentes, um recurso valioso e confiável. Existem muitas vantagens no uso dessa fonte de informação tecnológica, dentre elas, destaca-se a facilidade de acesso às bases de dados disponibilizadas gratuitamente na internet. Araújo (1981) descreve que, para o pesquisador, o documento de patente é "uma das mais ricas fontes de informação atualizada sobre o estado-da-arte, novas ideias e resolução de problemas. Assim, tudo isso pode conduzir a 
uma maior produtividade em suas atividades de pesquisa e desenvolvimento".

A patente constitui um título temporário de exclusividade concedido pelo Estado para exploração de uma nova tecnologia. Em contrapartida, essa concessão exige do titular a disponibilização da informação tecnológica da invenção, evitando o segredo da tecnologia e permitindo o acesso ao conhecimento.

Nesse contexto, este trabalho mostra o papel fundamental do profissional de informação como um ator indispensável para a realização dos estudos de prospecção, utilizando técnicas de estratégias de busca em bancos e bases de dados públicas e comerciais. Além disso, este estudo teve como objetivo ressaltar a importância da realização de um mapeamento de Prospecção Tecnológica como ferramenta indispensável para a cadeia produtiva do conhecimento. Para tanto, a metodologia de busca de anterioridade foi utilizada para verificar o estado atual de sistemas denominados de Túnel de diluição, sistemas estes que são utilizados para uma prévia diluição da exaustão veicular, com intuito de analisar os aerossóis provenientes da queima incompleta de combustíveis.

\section{0 uso de patentes em estudos de prospecção tecnológica}

A prospecção tecnológica, utilizando informações oriundas dos documentos de patentes, tem-se mostrado uma potente ferramenta e um instrumento bastante eficaz no apoio à tomada de decisão, tendo em vista - estado da arte disponível no seu conteúdo, que permite identificar tecnologias relevantes, parceiros, concorrentes no mercado, rotas tecnológicas, inovações, investimentos, processos, produtos, PD\&I, fusões e aquisições, dentre outras.

Um fator importante e que mostra a importância do uso das patentes, como fonte de informação tecnológica para a geração de mapas de conhecimento, reside no fato de que as bases de dados são padronizadas e com qualidade da informação, o que permite tratar estatisticamente volumes de dados com baixo risco de erros, que agrega valor ao conhecimento disponível.

A gestão do conhecimento vem como complemento às medidas de manter e criar vantagens competitivas. O diferencial de valor das organizações recai na sua capacidade de antecipar oportunidades e ameaças, bem como na apropriação do conhecimento tácito dos seus integrantes. Bens intangíveis passam a ser mais valorados, como patentes, know how e capital intelectual.

Para melhor entendimento sobre o uso da informação de patentes, vale salientar que o Brasil foi um dos 13 países a assinar a Convenção de Paris, em 1983, buscando sua inserção nos padrões internacionais de proteção aos direitos de propriedade intelectual. Porém, somente em 1996, a nova Lei 9.279 de patentes (BRASIL, 1996) eliminou a restrição da lei anterior, permitindo o patenteamento em áreas tecnológicas, como 
fármacos, medicamentos, produtos químicos, biotecnológicos e alimentos, dentre outras.

Após o período de privilégio, o invento cai em domínio público e pode derivar em novas apropriações do conhecimento tecnológico, com investimentos menores dos que os praticados na patente original. Essa tem se mostrado uma estratégia interessante de ampliação de mercado e melhoria da competitividade. Assim, países em desenvolvimento devem incentivar seu sistema nacional de inovação, na direção de combinar a absorção de tecnologia internacional e o desenvolvimento de capacitação interna, com legislação patenteária que estimule a difusão de inovações e que facilite a adaptação criativa de inovações às condições de demanda de cada país, contribuindo, assim, para o desenvolvimento de inovações de segunda geração, ou seja, de caráter incremental. A investigação e o monitoramento sistemático dos conteúdos dos documentos de patentes são, portanto, estratégias fundamentais na geração de conhecimentos.

\section{Prospecção tecnológica e sistemas de inteligência}

A prospecção tecnológica, no seu processo inicial, identifica um tema que necessita de monitoramento, isto é, monitorar o ambiente em busca de informação sobre aquele tema. A estratégia de busca deve ser estabelecida pelo profissional da informação, preferencialmente em parceria com o pesquisador. As fontes de informação são identificadas e ela é coletada, analisada e estruturada por um especialista na área do conhecimento do pesquisador demandante da busca. As principais fontes de informação em que se baseiam são as de natureza técnica e científica (artigos de periódicos, patentes, conferências, etc.).

Por outro lado, a inteligência competitiva é um processo sistemático de coleta, gestão, análise e disseminação da informação sobre os ambientes competitivos, concorrencial e organizacional, visando a subsidiar o processo decisório e atingir as metas estratégicas da organização. A inteligência competitiva constitui a coleta ética e o uso da informação pública disponível, sobre tendências, eventos e atores, fora das fronteiras da empresa. Para identificar as necessidades de informação da empresa é necessário: coletar sistematicamente a informação relevante e, em seguida, processá-la analiticamente, transformando-a em elemento para a tomada de decisão (COELHO, 2003).

O produto final da inteligência competitiva é a informação analisada, de interesse, para os tomadores de decisão, sobre o presente e futuro, na área de atuação da organização. Nos estudos de prospecção tecnológica, os sistemas de inteligência competitiva constituem o início e o fim do processo. O início, porque qualquer estudo prospectivo deve ter como base o monitoramento. O fim, porque a prospecção bem feita deve indicar as áreas prioritárias para o monitoramento sistemático, que é o que vai permitir à organização ter uma vantagem através do poder de antecipação. 


\section{Metodologia}

A metodologia de pesquisa, empregada com o objetivo de rastrear patentes de sistemas de diluição de aerossóis, provenientes da queima incompleta de combustíveis veiculares, consistiu em algumas determinações de busca. Primeiramente, foi definida a base de dados, que envolveu uma base internacional (European Patent Office - EPO) ${ }^{1}$, e outra nacional (Instituto Nacional de Propriedade Intelectual (INPI) ${ }^{2}$. Em seguida, foram determinadas palavras chave tanto em Português quanto em Inglês, para serem utilizadas nos processos de busca.

As palavras chave utilizadas nos bancos de dados nacionais foram: amostrador de volume constante, túnel de diluição, sonda de amostragem de partículas e amostrador de material particulado. Essas mesmas palavras chave, porém escritas em inglês, foram utilizadas no banco de dados internacional. Além dessas palavras chave, a pesquisa contou a com a presença de alguns recursos de pesquisa, como os operadores booleanos e os símbolos de truncatura, para que pudessem ajudar a encontrar o maior número possível de documentos. Mediante todos os resultados das buscas, foi realizada a seleção dos documentos de interesse através da análise de seus resumos, na qual, aqueles que eram foco da busca, foram analisados integralmente.

\section{Resultados e discussão}

De uma maneira geral, os resultados obtidos em ambos os bancos de dados selecionados, estão apresentados nos Gráficos 1 e 2. O Gráfico 1 apresenta o número de patentes encontradas no INPI em relação às palavras chave utilizadas na busca, juntamente com o recurso de pesquisa de truncatura. Dessa forma, somando o número de documentos encontrados com cada palavra chave, foram obtidos um total 19 documentos no banco de dados do INPI (Gráfico 1). Porém, ao analisar as patentes, pôde ser observado que o número total de documentos diferentes foi de apenas 13 documentos e que somente uma patente era relacionada ao tema da pesquisa.

No banco de dados internacional (EPO), com essas mesmas palavras chave em inglês, foram encontradas 388 patentes no total, um número bem mais significativo em relação aos documentos obtidos na base de dados nacional (INPI). Tal resultado pode ser explicado pelo fato de que, atualmente, no Brasil, são poucas as empresas e instituições que desenvolvem tecnologia na área de pesquisa do presente estudo. Assim, na Figura 2, estão apresentados os números de patentes encontradas. Dentre os documentos encontrados no EPO, apenas 18,56\% (72 patentes) eram relacionados ao tema de estudo. Além disso, somente 25 documentos puderam ser obtidos integralmente através do Download.

\footnotetext{
${ }^{1}$ Disponível em: <www.espacenet.com>. Acesso em 13 dez. 2012.

2 Disponível em: <www.inpi.gov.br>. Acesso em 13 dez. 2012.
} 
Gráfico 1 - Número de documentos encontrados no banco de dados do INPI

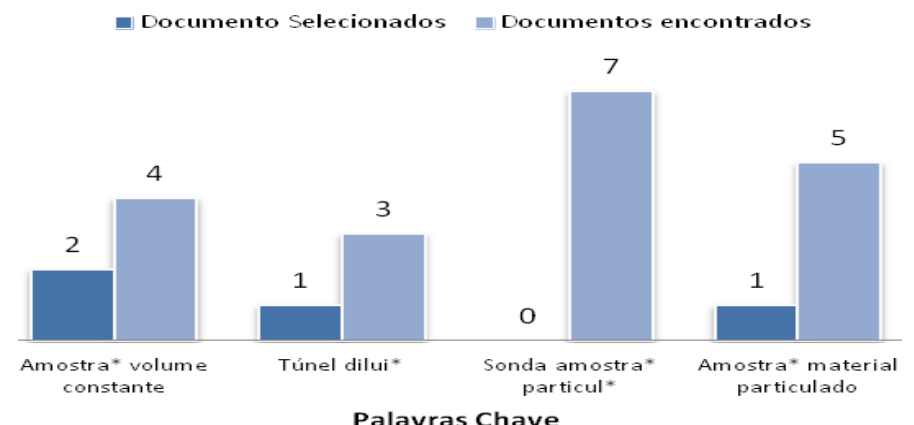

Fonte: Dados da pesquisa.

Gráfico 2 - Número de documentos encontrados no banco de dados do EPO

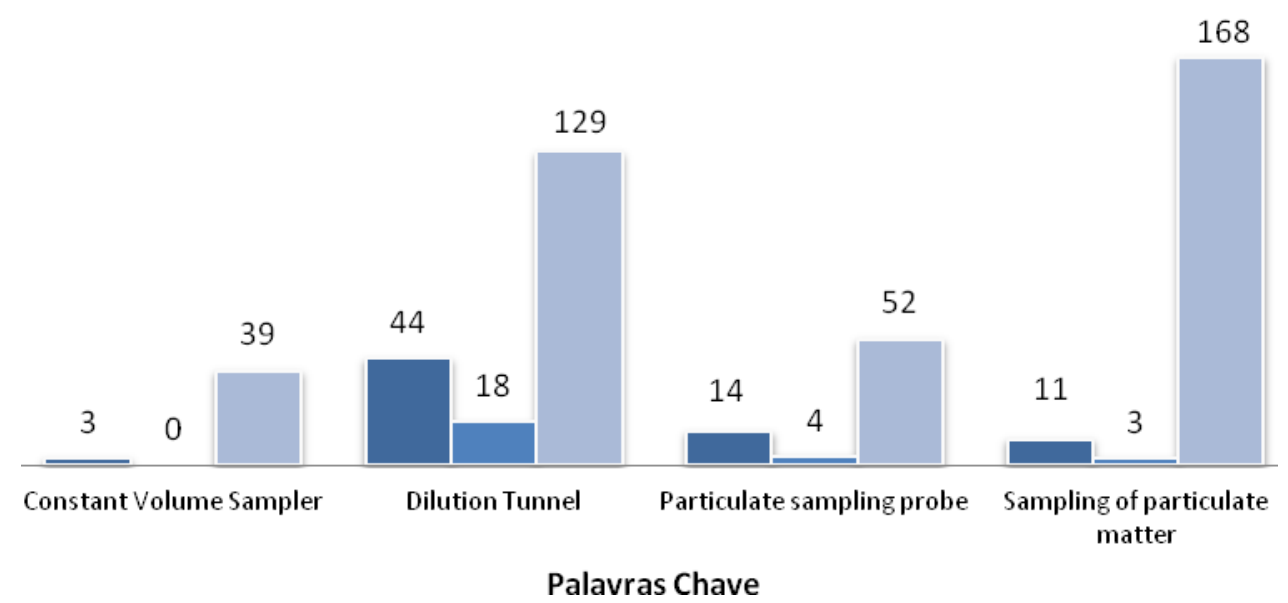

Fonte: Dados da pesquisa.

É importante destacar que a busca por anterioridade não consegue encontrar as patentes que estão em período de sigilo (18 meses). Assim, após a análise de todos os documentos obtidos em ambos os sítios de busca, descartando aqueles em duplicatas, de mesma família e outros que não tinham o foco do assunto de estudo, foram obtidos um total de 23 documentos nos portais do INPI e EPO.

Através do Gráfico 3a é possível observar a diferença significativa de documentos encontrados na base de dado nacional em relação à quantidade de patentes encontradas no EPO. Esse dado reflete a realidade atual do desenvolvimento nacional em relação ao foco do presente estudo. 
Gráfico 3 - (a) Balanço de patentes obtido na base de dados Nacional X Internacional (b) Número de patentes obtidas, agrupadas por Código CIP

— Patente Nacional Patente Internacional

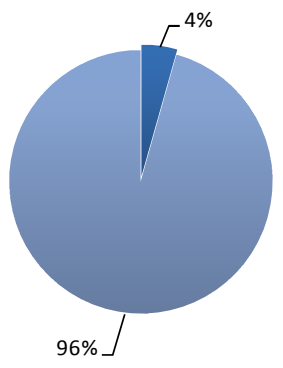

(a)

Fonte: Dados da pesquisa.

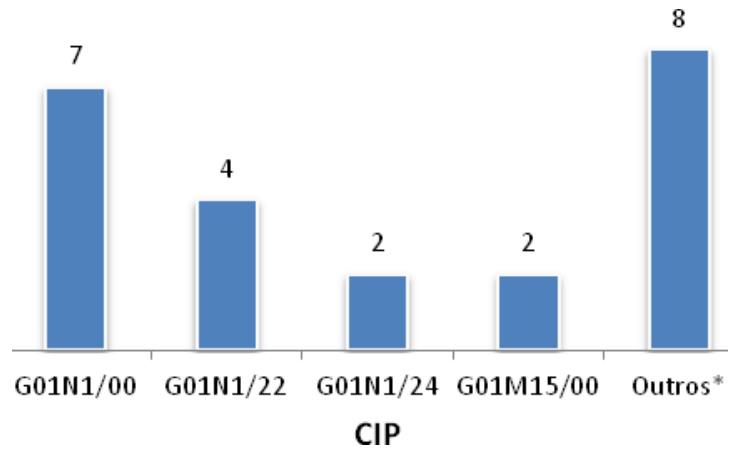

(b)

Em uma análise feita quanto à Classificação Internacional de Patentes (CIP), nos documentos obtidos integralmente, foi possível saber quais os códigos mais citados. Assim, observou-se que todos os documentos mantiveram um padrão quanto a CIP (Gráfico 3b). De acordo com as palavras chave utilizadas na busca, o código base mais utilizado foi o "G01N1", que está relacionado à "amostragem e preparo de espécies para análise".

Avaliando os documentos obtidos, quanto aos países que desenvolvem tecnologia para o tema em estudo, foi observado que os Estados Unidos, Japão e Alemanha são os grandes detentores da tecnologia relativa ao desenvolvimento de Túnel de diluição. No caso específico do Brasil, pode-se dizer que ainda são poucos os desenvolvimentos nesta área de estudo (Gráfico 4a). 
Gráfico 4 - (a) Número de patentes por país

(b) Classificação dos depositantes das patentes de túnel de diluição para determinação dos produtos oriundos da combustão incompleta da queima de combustíveis.

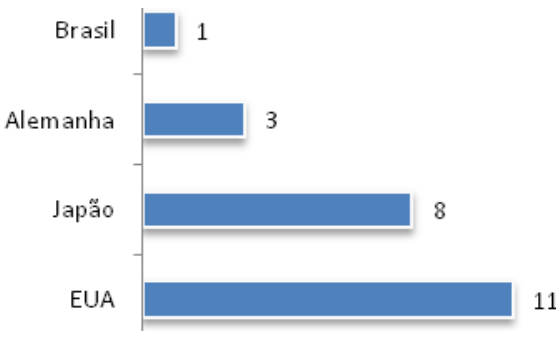

(a)

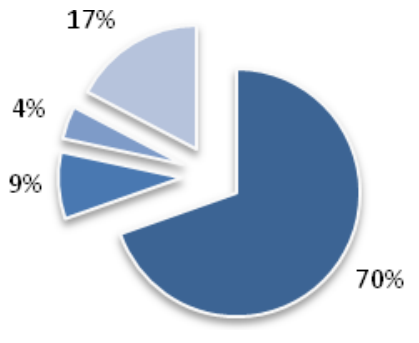

- Empresarial

- Acadêmico

- Governamental

Independente

Fonte: Dados da pesquisa.

Entre os depositantes de patentes, é possível identificar o percentual de documentos que foram depositados pela academia, por pessoas físicas, pelas corporações empresariais ou por instituições governamentais (QUINTELLA et al., 2011). Ao avaliar o perfil dos depositantes das tecnologias desenvolvidas para Túneis de diluição, pode-se observar que as empresas detêm a maior fatia da tecnologia, com $70 \%$ das patentes. As universidades e instituições governamentais possuem juntas $13 \%$ e pessoas físicas têm $17 \%$ das patentes (Gráfico $4 \mathrm{~b}$ ). Mediante os dados obtidos nesta prospecção, é possível inferir que grande parte da tecnologia que vem sendo desenvolvida para Túnel de diluição é oriunda de desenvolvimentos tecnológicos da linha empresarial e que poucos esforços vêm sendo feitos no setor acadêmico quanto ao assunto. Atualmente, estudos que necessitem da utilização de um túnel de diluição são realizados através da compra desse equipamento, que é bastante onerosa. Contudo, através do desenvolvimento inovador de um Túnel de diluição para aplicação em estudos de emissões veiculares, é possível mudar o cenário mundial e nacional quanto à detenção de tecnologia a respeito do tema. Além disso, o desenvolvimento de tal equipamento poderá reduzir consideravelmente o valor dele, se comparado com os que existem no mercado.

\section{Conclusões}

O processo de identificação, busca, análise e interpretação da informação, fato que cria conhecimento, permite uma sensível transformação e desenvolvimento daquele que incorpora tais conceitos e os pratica sistematicamente. Essa visão demonstra o potencial do estudo de prospecção tecnológica, principalmente no que se refere ao tratamento 
automático da informação, como ferramenta de apoio ao processo decisório, tendo vista a possibilidade de se ter uma visão sistêmica sobre as dinâmicas da pesquisa, desenvolvimento e inovação de um setor e/ou tecnologias, em curto espaço de tempo. Demonstra, também, o potencial de informação estratégica que as patentes podem oferecer no sentido de amparar um processo de gestão e monitoramento tecnológico, bem como de benchmarking de desenvolvimento tecnológico de empresas e de países.

A Prospecção tecnológica é de fundamental importância para área de desenvolvimentos de projetos. É uma ferramenta que deve ser utilizada por todos, pois influencia todo o processo de pesquisa $e$ desenvolvimento. Através do presente mapeamento de prospecção tecnológica, foi possível verificar a importância de se utilizar a Prospecção com foco na busca por anterioridade. Além disso, foi possível avaliar a viabilidade do desenvolvimento de um novo Túnel de diluição para estudos de emissões veiculares, identificando os pontos relevantes que podem diminuir o tempo de processo e identificar novas tecnologias. Assim, desenvolvimentos de "túnel de diluição" vêm sendo realizados mundialmente, em sua grande maioria, nos Estados Unidos, que é o país que detêm o maior número de depositantes, todos do setor empresarial.

Este estudo, também, pretende contribuir com a área de ciência da informação, apresentando a importância dos estudos de prospecção tecnológica com um serviço de alto valor agregado, bem como mostrando as possibilidades de ampliação do campo de atuação do profissional da informação, apresentando o quanto o uso efetivo do conhecimento explícito (seja em artigos, patentes, relatórios e outros) pode nortear a pesquisa, o desenvolvimento e a inovação tecnológica.

\section{Referências}

AGUIAR, A. C. Informação e atividades de desenvolvimento científico, tecnológico e industrial: tipologia proposta com base em análise funcional. Ciência da Informação, Brasília, v. 20, n. 1, p.7-15, jan./jun. 1991.

ALVARADO, R. U. A bibliometria no Brasil. Ciência da Informação, v. 13, n. 2, p. 91-105, 1984.

ANTUNES, A. M. S. Metodologia do estudo da trajetória de patenteamento da indústria de elastômeros através da elaboração de uma base de dados (1970-2000). In: WORKSHOP BRASILEIRO DE INTELIGÊNCIA COMPETITIVA E GESTÃO DO CONHECIMENTO, 3., 2002. Anais... São Paulo: KM Brasil, 2002.

ARAUJO, V. M. R. H. de. A patente como ferramenta da informação. Ciência da Informação, Brasília, v. 10, n. 2, p. 27-32, 1981.

BRASIL. Presidência da República, Casa Civil Subchefia para Assuntos Jurídicos. Regula direitos e obrigações relativos à propriedade industrial. Lei 9.279, de 14 de maio de 1996. Regula direitos e obrigações relativos à propriedade industrial. Diário Oficial da União, 15 maio 1996. 
AVL NORTH AMERICA INC.; SILVIS, W. M.; KREFT, N. SCHINDLER, W.; MAREK, G. Active Pulsation Cancellation device for diesel particulate sampling systems. US 6823748 B2. 12 de abril de 2002. 30 de novembro de 2004.

AVL NORTH AMERICA INC.; SILVIS, W. M.; KREFT, N.; MAREK, G. Particulate sampling probe and dilution tunnel. US 6857327 B2. 25 de novembro de 2003. 22 de fevereiro de 2005.

CANONGIA, C. et al. Foresight, inteligência competitiva e gestão do conhecimento: instrumentos para a gestão da inovação. Gestão \& Produção, v.11, n. 2, p. 231-238, mai./ago. 2004.

CASTELLS, M. A sociedade em rede. São Paulo: Paz e Terra, 1999.

COELHO, G. M. Prospecção tecnológica: metodologias e experiências nacionais e internacionais. Rio de Janeiro: INT, 2003.

DA SILVA, R. R. G. Procedimentos básicos de seleção de documentos para conversão digital: elementos de atualização profissional em setores de duas instituições públicas federais. Perspectivas em Ciência da Informação, v. 11, n. 3, p. 433-444, 2006.

GODET, M. A.; MONTI, R; MEUNIER F.; ROUBELAT, F. "caixa de ferramenta" da prospectiva estratégica. Lisboa: CEPES, 2000. (Caderno do CEPES).

INSTITUTO NACIONAL DA PROPRIEDADE INDUSTRIAL (INPI). 2011. Disponível em: <www.inpi.gov.br>. Acesso em: ago. 2011.

INSTITUTO NACIONAL DA PROPRIEDADE INDUSTRIAL (INPI). 2012. Disponível em: <http://pesquisa.inpi.gov.br/ipc/guide/br/CatchWord7.pdf>. Acesso em: fev. 2012.

KUPFER, D.; TIGRE, P. B. Modelo SENAI de prospecção: documento metodológico. Capítulo 2: prospecção tecnológica. In: ORGANIZACION INTERNACIONAL DEL TRABAJO CINTERFOR. Papeles de La Oficina Técnica. Montevideo: OIT/CINTERFOR, 2004. n. 14.

MAYERHOFF, Z. D. V. L. Uma análise sobre os estudos de prospecção tecnológica. Cadernos de Prospecção, v. 1, n. 1, p. 7-9, 2008.

QUINTELLA, C. M. et al. Prospecção tecnológica como uma ferramenta aplicada em ciência e tecnologia para se chegar à inovação. Revista Virtual de Química, v. 3, n. 5, p. 406-415, 2012.

\section{Patentes}

AVL NORTH AMERICA INC.; SILVIS, W. M.; KREFT, N. SCHINDLER, W.; MAREK, G. Active Pulsation Cancellation device for diesel particulate sampling systems. US 6823748 B2. 12 de abril de 2002. 30 de novembro de 2004. 
AVL NORTH AMERICA INC.; SILVIS, W. M.; KREFT, N.; MAREK, G. Particulate sampling probe and dilution tunnel. US 6857327 B2. 25 de novembro de 2003. 22 de fevereiro de 2005.

CATERPILLAR C/O LIELL, MCNEIL \& HARPER. GRAZE JR.; R. R.; RIEDLINGER, L. M.; RANDALL, J. K. Particulate sampling system and method of reducing oversampling during transients. US 2010/0101302 A1. 24 de outubro de 2008. 29 de abril de 2010.

CATERPILLAR INC. GRAZE, R. R.; PEORIA JR.; Dilution tunnel. EP 1367379 A1, 02 de abril de 2003. 03 de dezembro de 2003

CATERPILLAR INC.; GRAZE JR.; R. R. Gas sampling device and dilution tunnel used therewith. US 5058440. 4 de setembro de 1990. 22 de outubro de 1991.

CATERPILLAR INC; DICKSON, R. R.; GRAZE JR.; R. R. Method for controlling dilution air. US 6615677 B2 31 de outubro de 2001. 9 de setembro de 2003.

CATERPILLAR INC; DICKSON, R. R.; GRAZE JR; R. R. Exhaust gas particulate measuring system. US 7243559 B2. 24 de outubro de 2003. 17 de julho de 2007.

DAIMLER-BENZ AKTIENGESELLSCHAFT. SCHOBER, K.; GROLL, F. Device for sampling particles from the exhaust gases of a self-igniting internal combustion engine. US 4654058. 23 de maio de 1985. 31 de março de 1987.

HER MAJESTY THE QUEEN IN RIGHT OF CANADA AS REPRESENTED BY THE MINISTER OF THE ENVIRONMENT; HENDREN, F.; AINSLIE, $B$. Emission Sampling apparatus and method.US 6959590 B2. 24 de maio de 2001. 1 de novembro de 2005.

HINO JIDISHA KOGYO KABUSHIKI KAISHA; ICHIKAWA, J. Exhaust Gas measuring instrument. US 6460400. 18 de setembro de 1997. 8 de outubro de 2002.

HORIBA, LTD. YAMAGISHI, Y., ASAMI, T., OKUDA, S. Sampling Apparatus for exhaust gas. US 6370936 B1. 30 de abril de 1999. 16 de abril de 2002.

HORIBA, Ltd.; ISHIDA, K. Apparatus for measuring the soot particles contained in the exhaust gas emitted from diesel engines. US 4916384. 9 de dezembro de 1986. 10 de abril de 1990.

HORIBA, LTD.; YAMAGISHI, Y.; OHTSUKL, S. Exhaust Gas sampling Device. US 6615678 B2. 3 de agosto de 2001. 9 de setembro de 2003.

IVECO FIAT S.P.A. CONTROL SISTEM S.R.I.; D'ANGELO, L.; ROCCATELLO, G.; APPINO, A. Method and apparatus for extracting particulate from the exhaust gases of diesel engines. EP 0611962 A1. 18 de fevereiro de 1994. 24 de agosto de 1994. 
MITISUBISHI JIDOSHA KOGYO KABUSHIKI KAISHA. YAMASAKI, A.; TAKEDA Y.; ABE, S.; FUKANO, I. Multi-pipe flow-dividing dilution system. EP 0428850 A1, 28 de setembro de 1990. 29 de maio de 1991.

ONO SOKKI CO., LTD. SEKIYA, M.; FUKUSHIMA, S.; YOSHIMURA, Y.; IKEDA, T. Exhaust gas dilution apparatus. EP 1113257 A3. 22 de dezembro de 2000. 21 de abril de 2004.

THESSALONIKI ARISTOTLE UNIVERSITY; SAMARAS, Z. NTZIACHRISTOS, L. Diluter for exhaust gas sampling and method therefor. WO 2008/099224 A2. 14 de fevereiro de 2008. 21 de agosto de 2008.

UNIVERSIDADE FEDERAL DA BAHIA. TORRES, E. A.; DE ANDRADE, J. B.; GUARIEIRO, L. L. N.; PEREIRA, P. A. P. Túnel de Diluição para amostragem a volume constante de produtos de combustão incompleta emitidos na exaustão de motores veiculares. BR MU 8903123-7 U2. 23 de dezembro de 2009. 17 de janeiro de 2012. 